\section{Anestesiología}

Enero-Marzo 2021

Vol. 44. No. 1. pp 55-62

doi: $10.35366 / 97778$

\title{
Tratamiento del dolor en pacientes con quemaduras severas
}

\section{Pain management in patients with severe burns}

\author{
Dra. Evelyn Judith Cruz-Nocelo,* Dr. Víctor Hugo Zúñiga-Carmona, ${ }^{\ddagger}$ \\ Dra. María Concepción Serratos-Vázquez
}

Citar como: Cruz-Nocelo EJ, Zúñiga-Carmona VH, Serratos-Vázquez MC. Tratamiento del dolor en pacientes con quemaduras severas. Rev Mex Anest. 2021; 44 (1): 55-62. https://dx.doi.org/10.35366/97778

RESUMEN. Las quemaduras severas se caracterizan por inducir la respuesta metabólica más severa y sostenida respecto a cualquier otra forma de trauma. El tratamiento del dolor en estos pacientes es complejo. En esta revisión se integró el sustento científico disponible en la literatura actual desde un enfoque clínico-práctico en lo referente al tratamiento del dolor en el paciente con quemaduras; a su vez, se integraron dos esquemas representativos de la clasificación del dolor y de la analgesia multimodal para el paciente gran quemado. La analgesia multimodal es la técnica que se recomienda actualmente para el tratamiento del dolor por quemaduras; sin embargo, se requiere de investigaciones más amplias, en especial respecto a los adyuvantes analgésicos en pacientes quemados. Es probable que el estado de inflamación intensa y persistente que caracteriza a estos pacientes, que implica la reorganización genómica masiva del transcriptoma leucocitario, contribuya a la cualidad excepcional del dolor del paciente con lesiones por quemadura. El fundamento del manejo analgésico exitoso podría estar en la búsqueda de antiinflamatorios reguladores específicos para estos pacientes. Se requiere emprender el desarrollo de antiinflamatorios-analgésicos efectivos con propiedades que les permitan superar el reto de la alta variabilidad de los parámetros farmacológicos del paciente gran quemado.

ABSTRACT. Severe burns are characterized by inducing the most severe and sustained metabolic response than any other form of trauma. The treatment of pain in these patients is complex. In this review, the scientific support available in the current literature was integrated from a practical clinical approach in regard to the treatment of pain in the patient with burns; in turn, two representative schemes of the classification of pain and multimodal analgesia for the patient with major burns were integrated. Multimodal analgesia is the technique currently recommended for the treatment of burn pain; however, more research is required mainly with regard to analgesic adjuvants in burned patients. It is likely that the state of intense and persistent inflammation that characterizes these patients, which implies the massive genomic reorganization of the leukocyte transcriptome, contributes to the exceptional quality of the pain of the patient with burn injuries. The foundation of successful analgesic management could be in the search for specific regulatory anti-inflammatories for these patients. It is necessary to undertake the development of effective anti-inflammatoriesanalgesics with properties that allow them to overcome the challenge of the high variability of the pharmacological parameters of the patient with major burns.

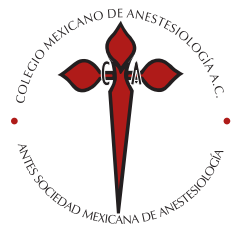

Palabras clave:

Quemaduras, manejo del dolor, inflamación, genómica.

Keywords:

Burns, pain management, inflammation, genomics.

* Servicio de Anestesiología del
Hospital de Traumatología.
‡ Centro Interdisciplinario para
el Estudio y Tratamiento del
Dolor y Cuidados Paliativos.

Unidad Médica de Alta Especialidad (UMAE) «Dr. Victorio de la Fuente Narváez», Instituto Mexicano del Seguro Social (IMSS). Ciudad de México, México.

Correspondencia:

Dra. Evelyn Judith Cruz-Nocelo Av. Pavo Real Ext. 60, Int. 21, Col. Las Alamedas, Atizapán, Estado de México, México. Teléfono: (52) 1551798 2170, (52) 5557473500 , ext. 25555 y 25556

E-mail: eve_412@hotmail.com

Recibido para publicación: 11-12-2018

Aceptado para publicación: 25-06-2019

\section{INTRODUCCIÓN}

L as quemaduras severas se caracterizan por inducir una $ـ$ respuesta metabólica severa y sostenida respecto a cualquier otra forma de trauma, por lo que son consideradas actualmente como la máxima expresión del trauma ${ }^{(1)}$. Según la American Burn Association, «Gran Quemado» o quemadura mayor se define como el paciente con una quemadura de más de $25 \%$ de superficie corporal total en adultos o de $20 \%$ en niños menores de 10 años y en adultos mayores de 40 años $^{(2)}$. Se estima que más de $80 \%$ de los pacientes con lesiones por quemadura describen su dolor con intensidad máxima e insoportable ${ }^{(3)}$.

El tratamiento del dolor en estos pacientes es complejo debido a que el dolor se define por el resultado del balance entre los mecanismos de nocicepción y de antinocicepción, bajo la influencia de un conjunto de factores neurobioquímicos, inflamatorios, metabólicos, genómicos, terapéuticos, psicológicos y socioafectivos sometidos al constante cambio. Existe evidencia de que el dolor es infratratado a pesar de las graves complicaciones inmediatas y de las consecuencias deletéreas a mediano y largo plazo que esto ocasiona ${ }^{(3,4)}$. En los primeros 
días posteriores a la quemadura, el tratamiento inadecuado del dolor puede generar un incremento en el consumo de oxígeno, favorecer una insuficiencia cardíaca y empeorar el estado de choque $^{(5-7)}$. Por otra parte, a mediano plazo los resultados de la terapia de rehabilitación se ven limitados por el dolor; la incidencia de dolor crónico y de desórdenes mentales como depresión, estrés postraumático y tendencia al suicidio se incrementan ${ }^{(4,8)}$. El objetivo de esta revisión es integrar el sustento científico disponible en la literatura actual desde un enfoque clínico-práctico en lo referente al tratamiento del dolor en el paciente con quemaduras severas.

\section{¿LA MÁXIMA EXPRESIÓN DEL TRAUMA TAMBIÉN CONLLEVA UNA MÁXIMA EXPRESIÓN DE DOLOR?}

Cuando la quemadura es leve se produce una respuesta inflamatoria local. En el caso de una quemadura severa se induce un síndrome de respuesta inflamatoria sistémica (SIRS), las citocinas producidas vierten hacia la circulación sistémica y son detectadas por el cerebro, en particular por el hipotálamo que envía señales para la liberación de catecolaminas y glucocorticoides por la glándula adrenal, lo que genera taquicardia, taquipnea y un incremento en la tasa metabólica ${ }^{(7,9)}$. Las quemaduras severas se caracterizan por inducir la respuesta metabólica más severa y sostenida respecto a cualquier otra forma de trauma, por lo que actualmente son consideradas como la máxima expresión del trauma ${ }^{(1)}$.

Xiao y colaboradores (2011) definen como «tormenta genómica» a la magnitud y la rapidez con la cual el transcriptoma leucocitario reorganiza y reprioriza sus patrones de expresión en respuesta al trauma severo. Los autores demostraron que la lesión tisular severa por trauma contuso y por quemadura indujo una respuesta genómica en la cual más de $80 \%$ del transcriptoma leucocitario se encontró alterado, afectando múltiples funciones y vías celulares. Estos cambios ocurrieron rápidamente dentro de las primeras cuatro a 12 horas y se prolongaron por días o semanas. En más de la mitad de los genes, los niveles de ARNm, después de 28 días en el trauma contuso y después de 90 días en pacientes quemados, aún no habían retornado a los niveles basales, lo cual es el reflejo de las alteraciones prolongadas que ocurren en el transcriptoma leucocitario del paciente con quemaduras severas ${ }^{(10)}$. Se sabe que existen varios mediadores inflamatorios que están relacionados con la transducción, la transmisión, la intromisión inflamatoria y el mantenimiento del dolor tanto a nivel periférico como a nivel central ${ }^{(11)}$. En consecuencia, es probable que este estado de inflamación persistente, que además se encuentra reorganizado genómicamente en tal magnitud, contribuya a la cualidad excepcional del dolor del paciente con lesiones por quemadura.

\section{CLASIFICACIÓN DEL DOLOR EN EL PACIENTE QUEMADO}

\section{Dolor inicial}

Minutos a horas después de la quemadura se liberan mediadores inflamatorios y vasoactivos que incrementan la permeabilidad capilar, causan vasodilatación y estasis vascular ${ }^{(12,13)}$. Inicialmente la respuesta es local, pero si la quemadura es grave también se manifestará de manera sistémica ${ }^{(14)}$. La microcirculación pierde su integridad capilar, habrá pérdida de líquido intravascular y fuga de proteínas plasmáticas hacia el intersticio, dando como resultado un edema masivo. Además, posterior a la reperfusión de los tejidos isquémicos se liberan radicales libres de oxígeno y otros metabolitos celulares tóxicos que causan disfunción de la membrana celular y mayor propagación de la respuesta inmunitaria ${ }^{(15-17)}$. En consecuencia, el componente inflamatorio del dolor es un factor predominante en esta fase.

El dolor inicial proviene de la estimulación y destrucción directa de los nociceptores. Las terminaciones nerviosas que fueron destruidas en su totalidad no trasmiten el estímulo doloroso; sin embargo, aquéllas que permanecieron intactas, pero expuestas son las que generaran dolor y alteraciones en la sensibilidad ${ }^{(5)}$.

A su vez, un componente neural en el área afectada estimula la liberación de norepinefrina por el sistema nervioso simpático seguido de una respuesta hormonal más lenta que eleva los niveles séricos de cortisol, epinefrina y aldosterona, esta respuesta al estrés depende de la magnitud de la lesión. En ocasiones, se puede manifestar una «analgesia inducida por el estrés» que consiste en la percepción de poco o nada de dolor en las horas inmediatas a la quemadura. Esto es regulado a nivel medular a través de vías inhibitorias descendentes y la liberación de endorfinas y otros neurotransmisores ${ }^{(5)}$. El objetivo principal de la terapia analgésica en esta fase es prevenir las consecuencias indeseables de esta respuesta al estrés como una insuficiencia adrenal ${ }^{(18)}$, un agravamiento del estado de choque y una insuficiencia cardíaca ${ }^{(5-7)}$.

Respecto al dolor inmediato a la quemadura, con frecuencia éste es descrito por los pacientes como dolor ardoroso o urente ${ }^{(19)}$, aunque comparte este descriptor con el dolor neuropático, en la actualidad no existe evidencia que sustente su tratamiento con neuromoduladores justificado en la indicación de aliviar ese síntoma en esta fase de la quemadura(5). También, es importante considerar que el incremento de las catecolaminas circulantes genera vasoconstricción a nivel renal y hepático; por lo tanto, el metabolismo y el aclaramiento de algunos fármacos disminuyen ${ }^{(20,21)}$. En consecuencia, el efecto de los fármacos analgésicos es impredecible; motivo por el que se sugiere utilizar opioides potentes de acción corta como la morfina en infusión intravenosa continua. 


\section{Dolor del proceso de recuperación}

La experiencia dolorosa durante el proceso de recuperación de las heridas por quemadura puede clasificarse en cuatro tipos de dolor: dolor de fondo, dolor irruptivo, dolor procedimental y dolor postoperatorio. Todos pueden estar presentes al mismo tiempo y cada uno de ellos requiere una evaluación independiente en términos de requerimiento analgésico ${ }^{(19,22)}$. En la Figura 1 se esquematizan los tipos de dolor y cada uno se explicará a continuación:

A. Dolor de fondo. Es el dolor persistente que ocurre a través del proceso de curación y dura hasta la reepitelización total de la herida; aunque se ha reportado que en ocasiones el dolor de fondo puede incrementarse justo después de que ésta se ha completado. Este dolor se presenta aun cuando el paciente está prácticamente inmóvil; se caracteriza por ser de duración prolongada, de naturaleza relativamente constante y de intensidad leve a moderada. Su tratamiento óptimo será consecuencia del sostenimiento de niveles séricos terapéuticos continuos de analgésicos efectivos ${ }^{(19)}$.

B. Dolor irruptivo. Se experimenta de manera espontánea, es el dolor impredecible y repentino con frecuencia asociado al movimiento, pero se presenta incluso cuando el paciente está relativamente inmóvil. Se caracteriza por ser de intensidad moderada a severa, se describe como punzante, quemante, como un «pinchazo» o «balazo». Se debe en particular a los mecanismos cambiantes del dolor a través del tiempo, pero también puede ocurrir cuando los niveles séricos terapéuticos de los analgésicos opioides bajan lo suficiente como consecuencia de un intervalo de dosificación inadecuado ${ }^{(19)}$.

C. Dolor procedimental. Es el dolor derivado de los procedimientos terapéuticos inherentes. Se describe como una sensación quemante intensa de característica punzante continua, pero disminuye con el tiempo. Con frecuencia se acompaña de un dolor penetrante intermitente intenso que aparece minutos a horas después de la ejecución de procedimientos que requieren la manipulación de tejido aún inflamado; por ejemplo: cambio de vendajes, desbridamiento de heridas, terapia física y ocupacional. Se considera uno de los dolores más intensos e induce ansiedad y distrés en el paciente. Éste puede ser tratado con analgésicos opioides potentes de acción corta vía intravenosa administrados durante el pico de hiperalgesia primaria inducido por el procedimiento ${ }^{(19,22)}$.

D. Dolor postoperatorio. Se considera uno de los dolores de mayor intensidad junto con el dolor procedimental. Con frecuencia la zona donadora de injerto es más dolorosa que el sitio injertado ${ }^{(19)}$. De acuerdo con las guías estadounidenses para el manejo del dolor postoperatorio es altamente recomendable organizar un plan de analgesia perioperatoria individualizado de acuerdo con la valoración y antecedentes

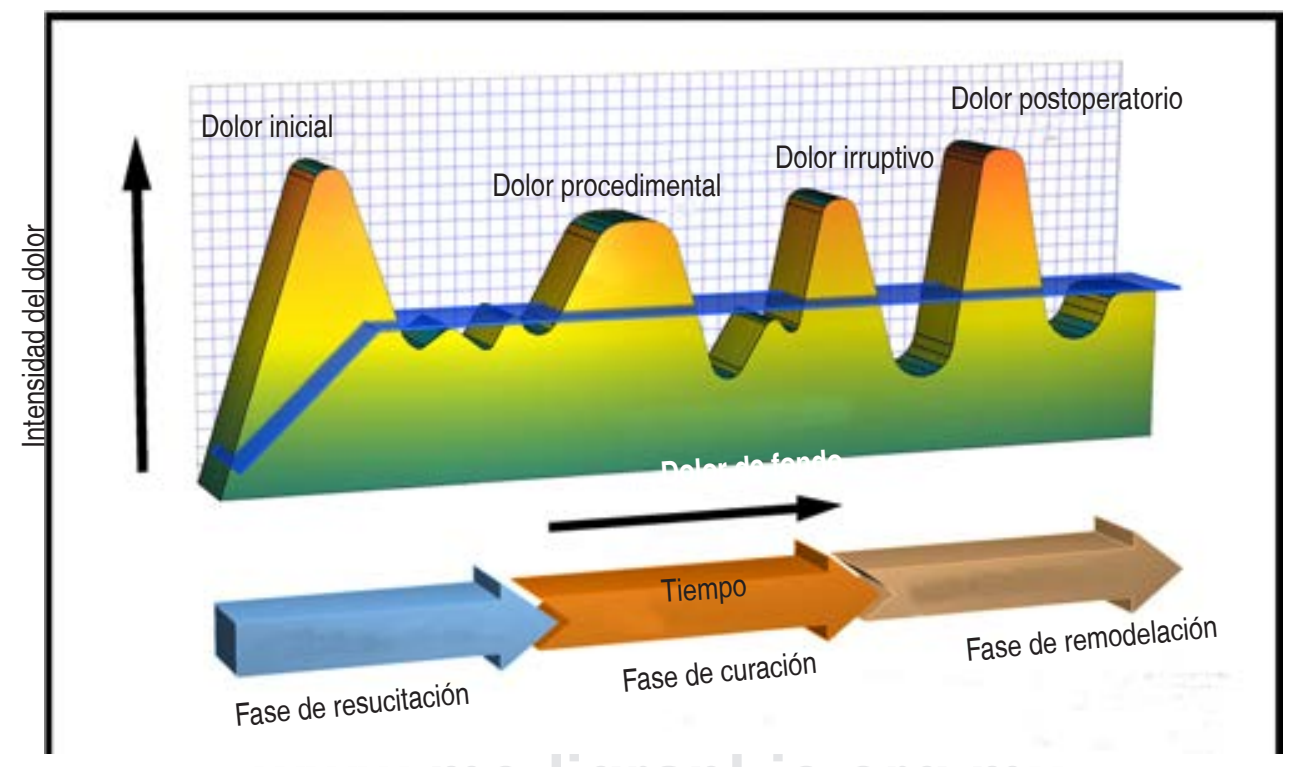

Tratamiento del dolor en pacientes con quemaduras severas

Cruz-Nocelo EJ, Zúñiga-Carmona VH, Serratos-Vázquez MC. 2021

Dibujo CAD por Cruz-Nocelo Miguel

Figura 1: Esquema de la clasificación del dolor en el paciente con quemaduras severas. Al establecer los objetivos del tratamiento se debe considerar que varios tipos de dolor pueden presentarse al mismo tiempo y cada uno de ellos requiere una evaluación independiente. Con la franja azul se representan los niveles séricos terapéuticos continuos de analgésicos opioides que permiten controlar el dolor de fondo. En la parte inferior se simbolizan las fases de recuperación de quemadura. 
del paciente, documentar el plan y sus objetivos en el expediente, explicar al paciente sus opciones y aclarar sus dudas; lo que disminuirá su ansiedad y mejorará los resultados de la terapéutica analgésica ${ }^{(23)}$. Las guías de recomendación para el dolor postoperatorio en el paciente quemado sugieren el empleo de la analgesia multimodal cuya base son los opioides para el dolor postoperatorio ${ }^{(8)}$. Aunque el empleo de opioides previo a la cirugía se asocia con mayor requerimiento postoperatorio de analgésicos, no hay evidencia para disminuir o descontinuar el opioide previo a la cirugía ${ }^{(23)}$. También, dentro del plan analgésico se sugiere incluir bloqueos regionales o locales; no obstante, se debe valorar bien al paciente y considerar los riesgos implícitos, ya que al ser un procedimiento invasivo en un paciente en estado inmunocomprometido conlleva cierto riesgo de infección ${ }^{(19)}$.

En adición a esta clasificación, el dolor por quemaduras puede dividirse en una combinación de síndromes dolorosos: dolor nociceptivo, dolor inflamatorio y dolor neuropático ${ }^{(24)}$.

\section{PRINCIPIOS GENERALES DEL TRATAMIENTO DEL DOLOR POR QUEMADURAS SEVERAS}

\section{Establecer objetivos del tratamiento analgésico}

Se deben tener concretamente determinados los propósitos del tratamiento analgésico más allá del control óptimo del dolor, por ejemplo: analgesia postoperatoria, rotación o disminución de la dosis del opioide, analgesia para terapia de rehabilitación, sedo-analgesia para paciente crítico, etcétera.

\section{Ubicar la fase de recuperación de la quemadura}

Las tres fases de la recuperación de una quemadura son: 1) fase aguda o fase de resucitación: incluye los primeros dos o tres días, 2) fase de curación: esta fase puede durar de semanas a meses, a veces años, tiempo durante el cual el paciente deberá someterse en múltiples ocasiones a procedimientos terapéuticos dolorosos, y 3) fase de rehabilitación o remodelación: es un período para completar el cierre de heridas y maduración de cicatrices; un tiempo para realizar terapia física y ocupacional agresiva para extender tejidos, prevenir contracturas y optimizar resultados funcionales ${ }^{(19,25)}$.

\section{Ubicar la fase de regulación metabólica de la quemadura}

Se han identificado dos patrones opuestos de regulación metabólica postquemadura: la fase de choque del quemado y la fase hipermetabólica. Ambas condiciones fisiopatológicas establecen cambios importantes a nivel de los compartimentos donde los fármacos, incluyendo los analgésicos, se distribuyen y se metabolizan ${ }^{(26)}$, por lo que es importante considerar que la farmacocinética y farmacodinamia son parámetros altamente variables en los pacientes con quemaduras severas ${ }^{(1,27)}$.

\section{Evaluación del dolor}

El McGill Pain Questionnaire, el Brief Pain Inventory y algunos otros sistemas de valoración se han empleado para evaluar la intensidad y otras dimensiones del dolor en pacientes quemados ${ }^{(22,25,28)}$. Independientemente de la escala de valoración que se utilice, lo más importante es asegurase de que el paciente comprende las instrucciones para expresar su dolor y no minimizar lo que describen. La evaluación del dolor requiere por lo menos de indagar acerca de la naturaleza, localización e irradiación del dolor, descripción del dolor, factores agravantes y atenuantes del dolor ${ }^{(29)}$.

A) Intensidad de dolor. Las quemaduras se clasifican de acuerdo con su profundidad, extensión y gravedad; no obstante, el dolor que se manifiesta no siempre es proporcional al resultado de estas valoraciones. El dolor es una experiencia única y personal, diferentes individuos pueden experimentar diferente sensación dolorosa aun con quemaduras similares, incluso un mismo individuo expresará intensidad variable de dolor durante las consecuentes fases de la evolución de su quemadura.

Se deben utilizar por lo menos dos escalas para valoración del dolor. La escala visual analógica ha sido previamente validada en pacientes quemados y consiste en que el paciente indicará al evaluador dónde detenerse al mover lentamente una marca sobre una línea de $10 \mathrm{~cm}$ desde una posición «sin dolor» hasta el máximo del «dolor insoportable», la longitud de la línea que el paciente indica es la medida y se registra en milímetros. Se sugiere completar la evaluación de la escala visual analógica con una escala verbal analógica del dolor. Si es el caso, se puede considerar el empleo de escalas especiales para pacientes mayores, niños o escalas conductuales. También, se recomienda valorar duración y horario del dolor. Los pacientes deben ser evaluados un mínimo de dos veces por día, pero de ser posible sería conveniente un registro por hora de la intensidad del dolor ${ }^{(22,28)}$.

B) Descriptores del dolor. El tipo de dolor se puede identificar de acuerdo con las características que el paciente describe, se sugiere un interrogatorio dirigido donde el paciente pueda expresar libremente su percepción de las molestias, pero si le es difícil este proceso es recomendable sugerir descriptores que el paciente pueda comprender ${ }^{(30)}$. Con frecuencia los pacientes refieren prurito, el mecanismo exacto de su origen no está muy claro, pero se piensa que involucra la liberación de histamina y prostaglandinas. El estímulo de los receptores de histamina es transmitido a través de receptores multimodales especializados presen- 


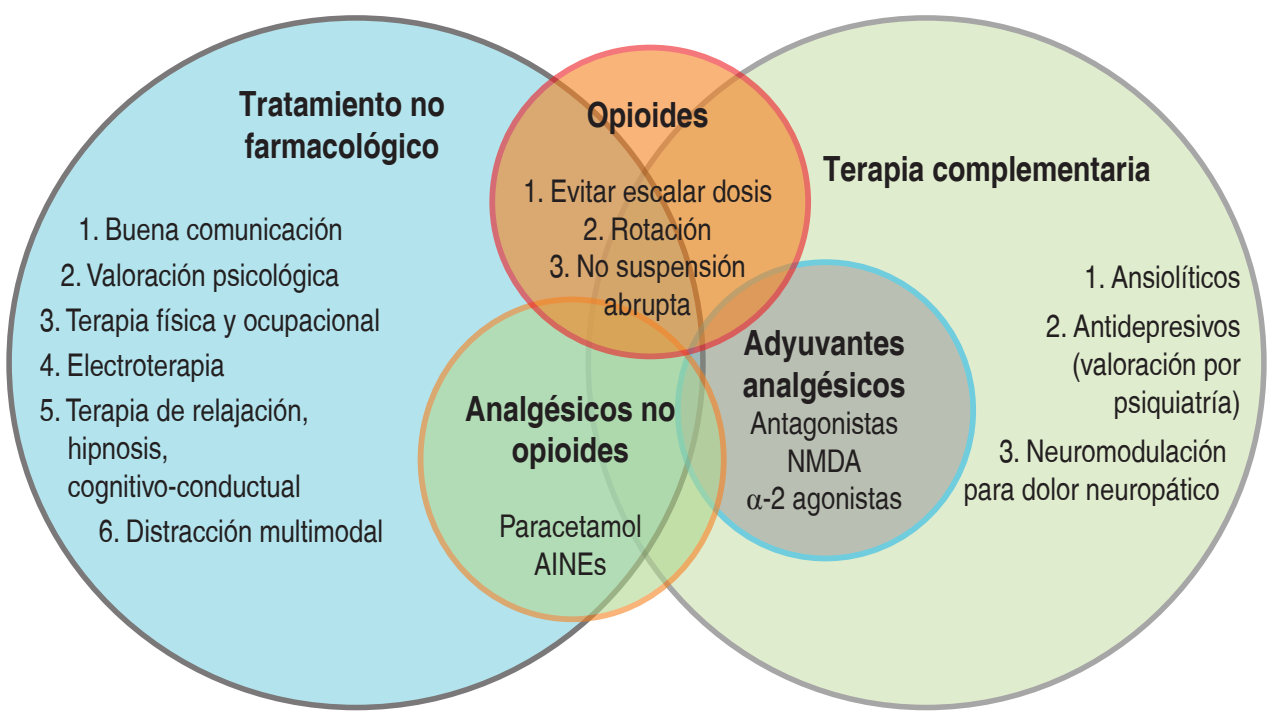

Tratamiento del dolor en pacientes con quemaduras severas

Cruz-Nocelo EJ, Zúñiga-Carmona VH, Serratos-Vázquez MC. 2021

Dibujo CAD por Cruz-Nocelo Miguel

Figura 2: Esquema de la analgesia multimodal recomendado para el tratamiento del dolor de moderado a severo del paciente con quemaduras severas. La base fundamental de este esquema son los opioides asociados a analgésicos no opioides (paracetamol y AINEs) y a adyuvantes analgésicos (principalmente antagonistas de los receptores NMDA). Un enfoque multidisciplinario que incluye métodos no farmacológicos y terapéutica complementaria es más efectivo en el control del dolor.

tes en la unión dermoepidérmica a fibras $\mathrm{C}$ amielínicas, hacen sinapsis a nivel medular y transmiten señales a través de neuronas de segundo orden del tracto espinotalámico anterolateral (vías moduladoras ascendentes y descendentes). El proceso supraespinal ocurre a nivel de la corteza cingulada anterior, del área motora suplementaria y del lóbulo parietal inferior. Por lo tanto, el prurito comparte la misma vía neuronal que el dolor y es considerado por algunos investigadores como una forma de dolor ${ }^{(5,25,30,31)}$. La gabapentina ha mostrado aliviar el prurito postquemadura ${ }^{(29)}$.

Además, se debe considerar que el daño severo en los nervios de la dermis profunda puede ocasionar al inicio un área relativamente insensible, pero posterior al proceso de regeneración neuronal, que se estima ocurre de cinco a seis semanas después de la quemadura, pueden surgir síntomas de dolor neuropático como resultado de una regeneración desordenada con sprouting a nervios vecinos, firing espontáneo o formación de neuroma. El dolor neuropático es descrito por los pacientes como dolor quemante, como puñaladas, disparos, piquetes, agujas y/o sensación de descarga eléctrica ${ }^{(22)}$. El dolor neuropático debe tratarse con neuromoduladores ${ }^{(29)}$.

C) Factores atenuantes o agravantes del dolor. Se sugiere siempre complementar el interrogatorio con la exploración física, así como la examinación de otras situaciones clínicas; por ejemplo: el dolor como resultado de un vendaje muy ajustado, edema tisular, infección de heridas, necrosis muscular en un síndrome compartimental, entre otras. También, valorar el estado metabólico, hidroelectrolítico y hemodinámico; la función hepática y renal; así como solicitar los estudios de laboratorio que se consideren necesarios ${ }^{(22)}$.

\section{TRATAMIENTO FARMACOLÓGICO}

Gamst-Jensen y colaboradores (2014) realizaron una evaluación de las guías de recomendación existentes para el manejo del dolor de los pacientes quemados; sólo se incluyeron cuatro guías dentro del estudio y de acuerdo a éste, las guías de Nueva Zelanda y de Estados Unidos resultaron evaluadas como altamente recomendables. Éstas coinciden en la recomendación de la analgesia multimodal para tratamiento del dolor de moderado a severo, en particular para el dolor postoperatorio, cuya base fundamental son los opioides asociados a analgésicos no opioides (paracetamol y AINEs) y a adyuvantes analgésicos ${ }^{(8)}$ como se esquematiza en la Figura 2. La analgesia multimodal se define como el empleo de una variedad de medicamentos y técnicas analgésicas que tienen diferentes mecanismos de acción con la finalidad de obtener un efecto sinérgico, mejor control del dolor y disminuir los efectos no deseados ${ }^{(23)}$. A continuación se describen algunas características importantes de los elementos de la analgesia multimodal. 


\section{Opioides}

Son analgésicos potentes y constituyen la piedra angular del tratamiento del dolor por quemaduras. Sin embargo, aunque proveen una analgesia excelente, no previenen el desarrollo de la sensibilización central y tienen efectos adversos bien conocidos como disfunción cognitiva, sedación, depresión respiratoria y constipación, pero su problemática principal radica en que su uso prolongado se acompaña de tolerancia, dependencia e hiperalgesia inducida por opioides ${ }^{(32)}$. Por esta razón, se sugiere la administración del opioide en infusión intravenosa continua para mantener concentraciones séricas terapéuticas empleando dosis menores y reservar las dosis en bolo para impregnación y como rescate para los picos de descontrol del dolor ${ }^{(19,22,32)}$. También se sugiere la rotación de opioides y evitar el escalamiento de la dosis; bajo una valoración periódica del dolor sería razonable incrementar la dosis mientras el dolor es intenso o cuando se espera que incremente y procurar reducir la dosis o rotar a opioide débil cuando mejore el control del dolor. El empleo de fármacos adyuvantes analgésicos permite reducir la dosis del opioide ${ }^{(32)}$. De igual manera, se recomienda evitar la suspensión abrupta de los opioides en los pacientes que han tenido tratamiento por tiempo prolongado ${ }^{(20)}$.

\section{Paracetamol}

Es recomendado para quemaduras severas como parte de una analgesia multimodal por su efecto sinérgico con los opioides $^{(29)}$. Puede ser administrado por períodos prolongados en forma segura, tiene pocas contraindicaciones y preserva la función renal incluso en el riñón bajo estrés. A dosis terapéuticas el paracetamol no modifica el flujo sanguíneo renal ni la tasa de filtración glomerular ${ }^{(33)}$.

\section{Antiinflamatorios no esteroideos}

Debido a las frecuentes complicaciones renales del paciente con quemaduras severas, el empleo de los AINEs está restringido; si las condiciones del paciente lo permiten se elegirán AINEs con escaso potencial nefro y hepatotóxico; de cualquier manera, su empleo se recomienda únicamente por períodos muy $\operatorname{cortos}^{(34)}$.

\section{Adyuvantes de la analgesia}

Los adyuvantes de la analgesia son fármacos de doble uso, comercializados para otras indicaciones diferentes al dolor, pero que son útiles como coanalgésicos cuando se agregan al tratamiento con opioides ${ }^{(35)}$. Entre ellos se encuentran la ketamina, la lidocaína endovenosa, los alfa-2-agonistas y la gabapentina, aunque se requiere de investigaciones más amplias respecto a su empleo en pacientes con quemaduras severas $^{(36-48)}$.

Algunos autores han sugerido protocolos de manejo del dolor para pacientes quemados, estos protocolos tienen gran utilidad como orientación; sin embargo, la principal recomendación consiste en individualizar y ajustar el tratamiento analgésico de acuerdo con cada evento emergente durante la recuperación del paciente ${ }^{(22,28)}$. Las modificaciones al plan analgésico se harán con base en el control del dolor y a la presencia de efectos secundarios, realizar un cambio a la vez permitirá evaluar el resultado e identificar sin dificultad al fármaco que podría originar efectos no deseados ${ }^{(23)}$. $\mathrm{Al}$ agregar un fármaco o incrementar la dosis se debe considerar que, a través de interacciones farmacológicas, también se pueden potencializar los efectos secundarios. A su vez, se sugiere evitar la indicación «por razón necesaria»; por el contrario, optar por una técnica intravenosa continua o analgesia controlada por el paciente en la que éste comprenda bien en qué momento debe y puede disponer de las dosis de rescate del opioide, o una combinación de ambas técnicas: la infusión intravenosa continua para tratar el dolor de fondo y los rescates para el dolor irruptivo y procedimental. Cabe mencionar que el manejo analgésico implica el tratamiento del dolor, de los síntomas asociados a éste (por ejemplo: ansiedad, depresión, insomnio y prurito) y de los efectos no deseados del esquema analgésico (por ejemplo: constipación, náusea, somnolencia e hiperalgesia inducida por opioides).

Los AINEs poseen propiedades que limitan su empleo continuo frente a la inflamación persistente en el paciente gran quemado. Es decir, actualmente no se cuenta con medicamentos capaces de modular este origen inflamatorio del dolor de manera efectiva y segura para estos pacientes. En su revisión McIntyre y colaboradores (2016) manifiestan la necesidad del desarrollo de nuevos analgésicos «hiperespecializados» para cada síndrome doloroso y fase del paciente gran quemado ${ }^{(24)}$.

De acuerdo con lo que se ha revisado e integrado en el presente trabajo, más que analgésicos específicos sin efectos secundarios; probablemente, considerando que uno de los principales estímulos de los nociceptores se origina a partir de la inflamación intensa y persistente que caracteriza la fisiopatología del gran quemado, el fundamento del manejo analgésico exitoso podría estar en la búsqueda de antiinflamatorios reguladores específicos capaces de modular la respuesta inflamatoria para que ésta sea suficiente para recuperar el estado de salud, pero no excesiva como para obtener los efectos deletéreos. Además, aún restaría que estos fármacos antiinflamatorios posean la capacidad de superar el reto de la alta variabilidad de los parámetros farmacológicos del paciente gran quemado. Por otra parte, evidentemente existe una brecha de conocimiento en términos de beneficio y seguridad de la aplicación de los adyuvantes analgésicos en pacientes quemados $^{(36-48)}$. Se requiere de la elaboración de un mayor 
número de estudios con nivel de evidencia superior que permita incrementar el grado de recomendación de la analgesia multimodal en el paciente con dolor por quemaduras severas.

\section{TERAPÉUTICA COMPLEMENTARIA Y TRATAMIENTO NO FARMACOLÓGICO}

Estudios previos en pacientes con quemaduras severas han mostrado la relación entre algunas respuestas psicoafectivas (como ansiedad, depresión, fatiga, trastorno de estrés, percepción de deformidad, entre otros) y mayores puntuaciones en escalas de intensidad del dolor ${ }^{(4)}$. En consecuencia, se recomienda un enfoque multidisciplinario para el tratamiento del dolor. Considerar las respuestas psicoafectivas del paciente y manejar oportunamente los desórdenes psicológicos y/o psiquiátricos debe ser una parte esencial del tratamiento individualizado ${ }^{(28,49)}$. Las benzodiazepinas son ansiolíticos que se emplean con frecuencia, aunque no son analgésicos permiten reducir la percepción del dolor en los pacientes con ansiedad importante. En algunos casos, la valoración psiquiátrica determinará si es necesario el empleo de antidepresivos. Algunos antidepresivos están indicados en el tratamiento del dolor neuropático ${ }^{(29)}$.

En adición a esto, se ha demostrado mayor efectividad en el control del dolor y reducción del distrés con el empleo de métodos no farmacológicos como terapias de relajación, dispositivos de distracción multimodales, terapias cognitivas, terapias conductuales, hipnosis, electroterapia, entre otros ${ }^{(50-52)}$.

\section{CONCLUSIONES}

Es posible que un método ordenado y basado en objetivos pueda ser el soporte de la estructura de la terapéutica analgésica individualizada para cada paciente. Ubicar la fase de regulación metabólica en la que se encuentre el paciente y la fase de recuperación de la quemadura, aunado a una evaluación práctica, metódica y completa, no sólo del dolor de manera aislada, sino del dolor como resultado de numerosas variables dinámicas dentro de un ser integrado, serán clave para el éxito en el tratamiento analgésico. Se requiere de investigaciones más amplias sobre el empleo de fármacos adyuvantes analgésicos en pacientes quemados.

En lo referente a quemaduras severas, se desconoce si la máxima expresión del trauma también conlleve una máxima expresión del dolor; sin embargo, es probable que este estado de inflamación intensa y persistente, que implica la reorganización genómica masiva del transcriptoma leucocitario, contribuya a la cualidad excepcional del dolor del paciente con lesiones por quemadura. Basados en esta teoría podríamos aseverar que, si se consiguiera el objetivo de modular la respuesta inflamatoria, como consecuencia directa el dolor inflamatorio característico y determinante del paciente gran quemado podría ser atenuado facilitando su tratamiento con medidas ordinarias; también, es probable que se evite el dolor persistente y el proceso que conduce al dolor crónico. De esta manera, el fundamento del manejo analgésico exitoso podría estar en la búsqueda de antiinflamatorios reguladores específicos con propiedades que les permitan superar el reto de la alta variabilidad de los parámetros farmacológicos del paciente gran quemado.

En esta revisión se presentó el sustento científico disponible en la literatura actual desde un enfoque clínico-práctico en lo referente al tratamiento del dolor en el paciente con quemaduras; a su vez, se integraron dos esquemas representativos de la clasificación del dolor y de la analgesia multimodal para el paciente gran quemado.

\section{AGRADECIMIENTOS}

A Miguel Cruz-Nocelo, Ingeniero en Mecatrónica, por la realización de los dibujos CAD.

\section{REFERENCIAS}

1. Pereira CT, Murphy KD, Herndon DN. Altering metabolism. J Burn Care Rehabil. 2005;26:194-199.

2. Kaiser HE, Meerim CK, Sharar SR, Olivar HP. Advances in perioperative and critical care of the burn patient. Adv Anesth. 2013;31:137-161.

3. Perry S, Heidrich G, Ramos E. Assessment of pain in burned patients. J Burn Care Rehabil. 1981;2:322-326.

4. Esfahlan AJ, Lotfi M, Zamanzadeh V, Babapuor J. Burn pain and patients' responses. Burns. 2010;36:1129-1133.

5. Norman AT, Judkins KC. Pain in the patient with burns. Contin Educ Anaesth Crit Care Pain. 2004;4:57-61.

6. Serratos-Vázquez MC. Manejo de dolor en el paciente con trauma. Rev Mex Anest. 2012;35 Supl 1:123-129.

7. Williams FN, Jeschke MG, Chinkes DL, Suman OE, Branski LK, Herndon DN. Modulation of the hypermetabolic response to trauma: temperature, nutrition, and drugs. J Am Coll Surg. 2009;208:489-502.
8. Gamst-Jensen H, Nygaard-Vedel P, Lindberg-Larsen VO, Egerod I. Acute pain management in burn patients: Appraisal and thematic analysis of four clinical guidelines. Burns. 2014;40:1463-1469.

9. Greenhalgh DG. Sepsis in the burn patient: a different problem than sepsis in the general population. Burns Trauma. 2017;5:23.

10. Xiao W, Mindrinos MN, Seok J, Cuschieri J, Cuenca AG, Gao H, et al. A genomic storm in critically injured humans. J Exp Med. 2011;208:2581-2590.

11. De Lille FR. Fisiopatología del dolor. En: Guevara LUM, editor. Dolor por especialidades. México: Corinter; 2008. pp. 19-39.

12. Beushausen T, Mucke K. Anesthesia and pain management in pediatric burn patients. Pediatr Surg Int. 1997;12:327-333.

13. Wolf SE, Debroy M, Herndon DN. The cornerstones and direction of pediatric burn care. Pediatr Surg Int. 1997;12:312-320.

14. Silvestre-Pérez MA, Matoses-Jaén MS, Peiró-Tudela MC, López-Navarro AM, Tomás-Braulio J. Anestesia y reanimación 
para el gran quemado pediátrico. Rev Esp Anestesiol Reanim. 2004;51:253-267.

15. Demling RH. The burn edema process: current concepts. J Burn Care Rehabil. 2005;26:207-227.

16. Ahrns KS. Trends of burn resuscitation: Shifting the focus from fluids to adecuate end point monitoring, edema control, and adyuvant therapies. Crit Care Nurs Clin North Am. 2004;16:75-98.

17. Latenser BA. Critical care of the burn patient: the first 48 hours. J Crit Care Med. 2009;37:2819-2826.

18. Fuchs PCh, Groger A, Bozkurt A, Johnen D, Wolter T, Pallua N. Cortisol in severely burned patients: investigations on disturbance of the hypothalamic-pituitary-adrenal axis. Shock. 2007;28:662-667.

19. Summer GJ, Puntillo KA, Miaskowski C, Green PG, Levine JD. Burn injury pain: the continuing challenge. J Pain. 2007;8:533-548.

20. Anderson TA, Fuzaylov G. Perioperative anesthesia management of the burn patient. Surg Clin North Am. 2014;94:851-861.

21. Jeschke MG. The hepatic response to thermal injury: Is the liver important for postburn outcomes? Mol Med. 2009;15:337-351.

22. Richardson P, Mustard L. The management of pain in the burns unit. Burns. 2009;35:921-936.

23. Chou R, Gordon DB, De Leon-Casasola OA, Rosenberg JM, Bickler S, Brennan T, et al. Management of postoperative pain: A clinical practice guideline from the American Pain Society, the American Society of Regional Anesthesia and Pain Medicine, and the American Society of Anesthesiologists' Committee on Regional Anesthesia, executive committee, and administrative council. J Pain. 2016;17:131-157.

24. McIntyre MK, Clifford JL, Maani CV, Burmeister DM. Progress of clinical practice on the management of burn-associated pain: Lessons from animal models. Burns. 2016;42:1161-1172.

25. Falder S, Browne A, Edgar D, Staples E, Fong J, Rea S, et al. Core outcomes for adult burn survivors: A clinical overview. Burns. 2009;35:618-641.

26. Jeschke MG, Gauglitz GG, Kulp GA, Finnerty CC, Williams FN, Kraft $\mathrm{R}$, et al. Long-term persistence of the pathophysiologic response to severe burn injury. PLoS One. 2011;6:e21245.

27. Blanchet B, Jullien V, Vinsonneau C, Tod M. Influence of burns on pharmacokinetics and pharmacodynamics of drugs used in the care of burn patients. Clin Pharmacokinet. 2008;47: 635-654.

28. Yang HT, Hur G, Kwak IS, Yim H, Cho YS, Kim D, et al. Improvement of burn pain management through routine pain monitoring and pain management protocol. Burns. 2013;39:619-624.

29. Retrouvey H, Shahrokhi S. Pain and the thermally injured patient- a review of current therapies. J Burn Care Res. 2015;36:315-323.

30. Álvarez ET. Semiología del dolor. Iatreia. 2002;15:200-206.

31. Zachariah JR, Rao AL, Prabha R, Gupta AK, Paul MK, Lamba S. Post burn pruritus-A review of current treatment options. Burns. 2012;38:621-629.

32. Holtman JR Jr, Jellish WS. Opioid-induced hyperalgesia and burn pain. J Burn Care Res. 2012;33:692-701.

33. Covarrubias-Gómez A, González García JL, Betancourt-Sandoval JA, Mendoza-Reyes JJ. El dolor agudo perioperatorio y el paracetamol: una visión basada en la evidencia. Rev Mex Anest. 2013;36:47-55.

34. Tamayo VAC. Analgésicos no opiáceos. Inhibidores de la ciclooxigenasa. En: Guevara LUM, editor. Dolor por especialidades. México: Corinter; 2008. pp. 735-751.

35. Allende PA, Acosta EA, Aguilar LS, Alcorta GAG, Arzate MCE, Ascencio HL, et al. Consenso Mexicano de Dolor por Cáncer. Gaceta Mexicana de Oncología. 2016;15 Supl 1:3-34.
36. McGuiness SK, Wasiak J, Cleland H, Symons J, Hogan L, Hucker T, et al. A systematic review of ketamine as an analgesic agent in adult burn injuries. Pain Med. 2011;12:1551-1558.

37. De Oliveira CM, Issy AM, Sakata RK. Intraoperative intravenous lidocaine. Rev Bras Anestesiol. 2010;60:325-333.

38. Muth-Selbach U, Hermanns H, Stegmann JU, et al. Antinociceptive effects of systemic lidocaine: Involvement of spinal glycinergic system. Eur J Pharmacol. 2009;613:68-73.

39. Wasiak J, Spinks A, Costello V, Ferraro F, Paul E, Konstantatos A, et al. Adjuvant use of intravenous lidocaine for procedural burn pain relief: A randomized double-blind, placebo-controlled, cross-over trial. Burns. 2011;37:951-957.

40. Wasiak J, Mahar PD, McGuinness SK, et al. Intravenous lidocaine for the treatment of background or procedural burn pain. Cochr Datab Syst Rev. 2014;CD005622.

41. Helander EM, Menard BL, Harmon CM, Homra BK, Allain, AV, Bordelon GJ, et al. Multimodal analgesia, current concepts, and acute pain considerations. Curr Pain Headache Rep. 2017;21:3.

42. Blaudszun G, Lysakowski C, Elia N, Tramèr MR. Effect of perioperative systemic $\alpha 2$ agonists on postoperative morphine consumption and pain intensity: systematic review and meta-analysis of randomized controlled trials. Anesthesiology. 2012;116:1312-1322.

43. Asmussen S, Maybauer DM, Fraser JF, Jennings K, George S, Maybauer MO. A meta-analysis of analgesic and sedative effects of dexmedetomidine in burn patients. Burns. 2012;39:625-631.

44. Cuignet O, Pirson J, Soudon O, Zizi M. Effects of gabapentin on morphine consumption and pain in severely burned patients. Burns. 2007;33:81-86.

45. Gray P, Williams B, Cramond T. Successful use of gabapentin in acute pain management following burn injury: a case series. Pain Med. 2008;9:371-376.

46. Rimaz S, Alavi CE, Sedighinejad A, Tolouie M, Kavoosi S, Koochakinejad L. Effect of gabapentin on morphine consumption and pain after surgical debridement of burn wounds: a double-blind randomized clinical trial study. Arch Tauma Res. 2012;1:38-43.

47. Wibbenmeyer L, Eid A, Liao J, Heard J, Horsefield A, Kral L, et al. Gabapentin is ineffective as an analgesic adjunct in the immediate postburn period. J Burn Care Res. 2014;35:136-142.

48. Yuxiang L, Lingjun Z, Lu T, Mengjie L, Xing M, Fengping S, et al. Burn patients' experience of pain management: a qualitative study. Burns. 2012;38:180-186

49. Andrews RM, Browne AL, Wood F, Schug SA. Predictors of patient satisfaction with pain management and improvement 3 months after burn injury. J Burn Care Res. 2012;33:442-452.

50. Provençal SC, Bond S, Rizkallah E, El-Baalbaki G. Hypnosis for burn wound care pain and anxiety: a systematic review and meta-analysis. Burns. 2018:44:1870-1881.

51. Scheffler M, Koranyi S, Meissner W, Strauß B, Rosendahl J. Efficacy of non-pharmacological interventions for procedural pain relief in adults undergoing burn wound care: A systematic review and meta-analysis of randomized controlled trials. Burns. 2018;44:1709-1720.

52. Pérez-Ruvalcaba I, Sánchez-Hernández V, Mercado-Sesma AR. Effect of a combined continuous and intermittent transcutaneous electrical nerve stimulation on pain perception of burn patients evaluated by visual analog scale: a pilot study. Local Reg Anesth. 2015;8:119-122. 